\title{
Curie Point Pyrolysis Gas Chromatography
}

\section{Journal Article}

Author(s):

Bühler, Ch.; Simon, W.

Publication date:

1970

Permanent link:

https://doi.org/10.3929/ethz-b-000422789

Rights / license:

In Copyright - Non-Commercial Use Permitted

Originally published in:

Journal of Chromatographic Science 8(6), https://doi.org/10.1093/chromsci/8.6.323 
phase.

The results of these preliminary experiments furnish useful guidance for the development of this method. In order to shorten the time required for the separation at a high efficiency, faster mixing and therefore higher gyration speed $\omega$ is necessary. Once $\omega$ is determined, the optimal condition for both phase volume ratio and interfacial area can be satisfied by angle $\alpha$ for a given column. Thus, the major requirements for the design of the apparatus will be finely adjustable radius of gyration, well-balanced system, and uniform speed of gyration to minimize carry-over of the stationary phase. Though high speeds of gyration should be applied, the resultant centrifugal force is as small as several $\mathbf{g}$ values by selecting the proper radius $r$ for the optimal angle $\alpha$.

When the above requirements are fulfilled, the method will greatly extend the capability of gyration L.CCC. Because of the evenly distributed gyration effect and its low column pressure, the applicable column length is almost limitless. The symmetrical cir- cular stirring of the liquids enables a high partition efficiency of a few $\mathrm{mm}$ in HETP. The analytical application is possible with a fine column by the use of the centrifugal force to prevent plug flow, and the preparative scale-up is easily achieved on a wider column without loss of efficiency. With combination of a high flow rate and a fast gyration speed, time requirement for separation may be reduced to the order of 1 second per transfer corresponding to the conventional liquid extraction techniques.

\section{Acknowledgments}

We are indebted to $\mathrm{Mr}$. H. Chapman for engineering works and to Miss June Friedlander for preparation of manuscript.

\section{Manuscript received March 20, 1970}

Presented at the Sixth International Symposium on Advances in Chromatography held in Miami Beach, Florida, June 2-5, 1970.

\title{
Curie Point Pyrolysis Gas Chromatography
}

\author{
by Ch. Bühler and W. Simon, Swiss Federal Institute of Technology, \\ Laboratory for Organic Chemistry, Zurich, Switzerland
}

\begin{abstract}
In Curie Point pyrolysis gas chromatography a ferromagnetic conductor in contact with the sample is heated inside a low volume glass capillary which is inserted into an if coil with the carrier gas flowing through the capillary. The intrinsic properties of the conductor permit a self-control of the temperature to the Curie Point. The optimum wire diameter for a rapid warm-up is a function of the if frequency. In order to have a fast temperature drop after cutting the rf field, small wire diameters are preferable. For such conductors relatively high frequencies are inherently necessary for a fast stabilization to the Curie Point. So far no differences in the self-controlled end-temperatures have been detected for wires of different origin.
\end{abstract}

In pyrolysis gas chromatography, the samples to be studied are thermally fragmented in a stream of a carrier gas and the reaction products are passed directly into a gas chromatographic column where they are separated. There are two extreme possibilities for the application of this technique (1):

a) The pyrolysis gas chromatogram is simply used as a "fingerprint" of the starting material.

b) Correlations between the structure of the fragments and the structure of the starting material are worked out and used for structural elucidation, identification and quantitative analysis.

There is by now general agreement that adequate long term and inter-laboratory reproducibility in thermal fragmentation can only be achieved by using a highly reproducible temperature-time profile for the fragmentation process (1-5). For nonvolatile or slightly volatile compounds the application of a square-wave temperature-time profile to the highly diluted sample

1. Simon, W., VI. Symposium über Gas-Chromatographie, Berlin, May 14-18, 1968.

2. Levy, R. L., and Fanter, D. L., Anal. Chem. 41, 1465 (1969).

3. Levy, R. L., J. Gas Chromatog. 5, 107 (1967).

4. Levy, R. L., Chairman of a panel discussion in "Gas Chromatography 1968," C. L. A. Harbourn, ed., Institute of Petroleum, London, 1969.

5. Farré-Rius, F., and Guiochon, G., Anal. Chem. 40, 998 (1968) 

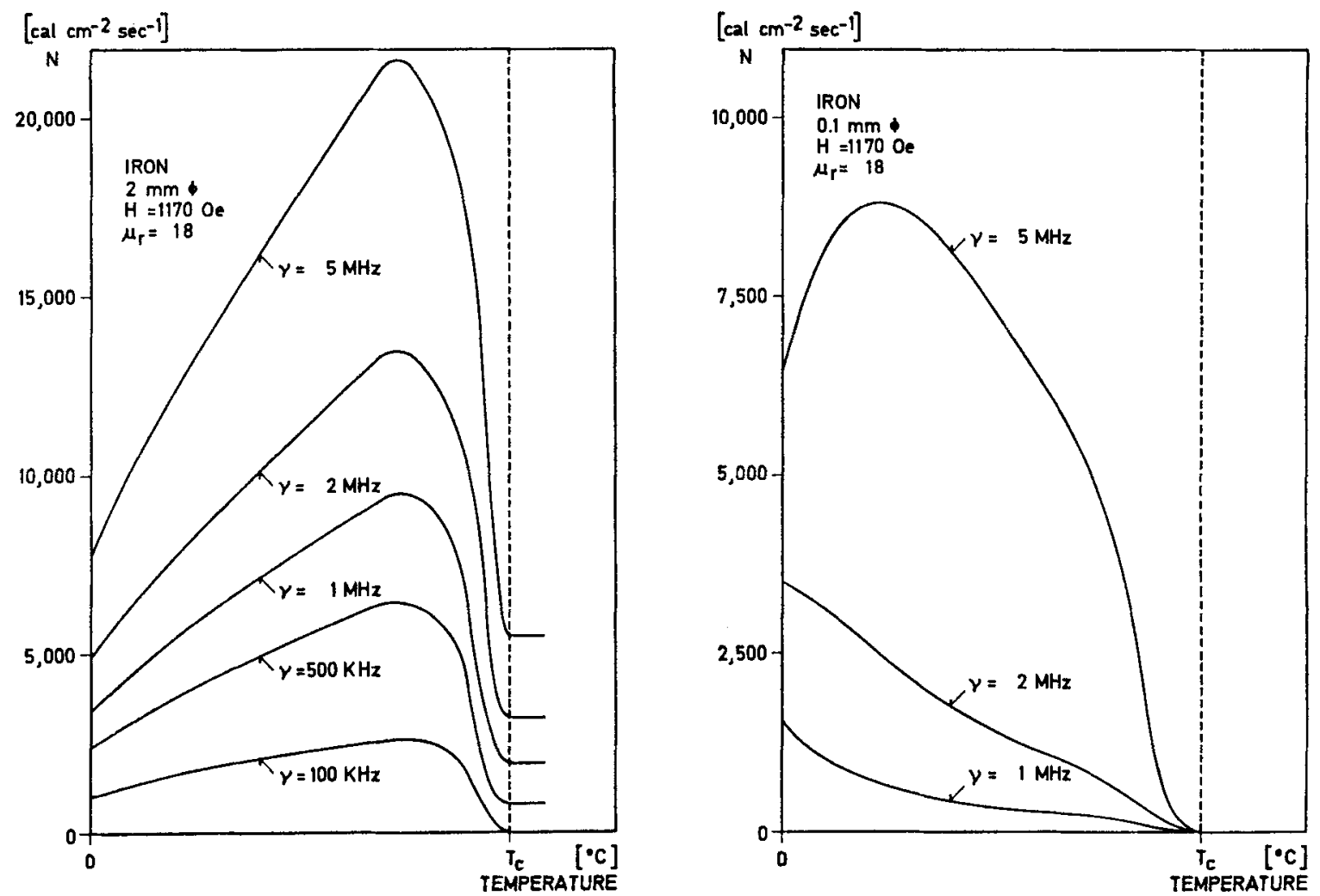

Figure 1. Temperature dependence of the power consumption of iron conductors in a high frequency induction field of 1170 Oe as a function of the oscillator frequency.

seems to be ideal (1-15)). We have shown earlier $(6,7)$ that a fast and reproducible warm-up of samples in contact with cylindric ferromagnetic conductors can be obtained by high frequency induction heating. Conductors of $0.5 \mathrm{~mm}$ diameter can reach the Curie temperature of the ferromagnetic material in under $30 \cdot 10^{-3}$ sec (7). Although the use of high frequency induction heating in pyrolysis had been suggested earlier by $\mathbf{H}$. Szymanski et al., (16, cf 17) the possibility of limiting the temperature to the Curie Point and therefore achieving an inherently well-defined end temperature was not realized at that time.

The power consumption $\mathrm{N}$ [cal $\left.\mathrm{cm}^{-2} \mathrm{sec}^{-1}\right]$ per unit surface of a ferromagnetic conductor located along the axis inside a high-frequency induction coil $(7,18-20)$ is given by

$$
\mathrm{N}=2 \pi \sqrt{2} \mathrm{H}^{2} \rho(1 / \mathrm{s}) \mathrm{F}(\mathrm{r}, \mathrm{s}) \quad \text { Eq. } 1
$$

where

$$
\mathbf{s}=\sqrt{\rho / \pi \gamma \mu_{0} \mu_{\mathrm{r}}}
$$

H: Magnetic field inside the coil

$r$ : Radius of the conductor

$\rho$ : Specific electric resistance of the conductor

s: Skin depth of the eddy current

F: Function of $\mathbf{r} / \mathrm{s} ; \lim \mathrm{F}(\mathrm{s}, \mathbf{r}) \frac{\mathrm{r}}{\mathrm{s}} \rightarrow \infty=\frac{1}{\sqrt{2}}$

$\mu_{\mathrm{r}}$ : Relative permeability $(>1$ for ferromagnetic conductors below the Curie temperature $T_{e}, \sim 1$ for non-ferromagnetic conductors)

$\mu_{0}=4 \pi \cdot 10^{-9} \mathrm{~V} \mathrm{sec} / \mathrm{Acm}$

$v$ : Frequency of the oscillator.

The relative permeability $\mu_{\mathrm{r}}$ of a ferromagnetic conductor suddenly drops when it reaches the Curie Point
(19). This usually results in a sudden increase in the skin depth $s$ so that $1 / s$ and $F(s, r)$ become smaller and the power consumption of the conductor decreases. A further increase in temperature may therefore be inhibited. However, it is important to realize that $\mu_{\mathrm{r}}$ is not only a function of the temperature but also of the magnetic field $H$. To illustrate the effect of the different parameters the power consumption for situations of practical importance (see Figures 1-4 and Table I)

6. Giacobbo, H., and Simon, W., Pharm. Acta Helv. 39, 162 (1964); Festschrift zum 60. Geburtstag von Jacob Büchi (X. Perlia, ed.), Verlag Schweizer Apothekerverein, Zürich, 1963, S. 199.

7. Simon, W., and Giacobbo, H., Chemie-Ing.-Tech. 37, 709 (1965).; Angew. Chem. Internat. Edit. 4, 940 (1965).

8. Levy, R. L., Chromatog. Rev. 8, 48 (1966).

9. Perry, S. G., J. Gas Chromatog. 5, 77 (1967).

10. Noffz, D., and Pfab, W., Z. Anal. Chem. 228, 188 (1967)

11. Noffz, D., Pfab, W., and Benz, W., GDCh-Hauptversammlung, Fachgruppe Analytische Chemie, Berlin, 1967.

12. Brauer, G. M., J. Polymer Sci. Part C 8, 3 (1965).

13. Coupe, N. B., and McKeown, M. C., Pye Unicam Chromatography Bulletin 2, (4), 8 (1968).

14. Willmott, F. W., J. Chromatog. Sci. 7, 101 (1969).

15. Audebert, R., Ann. Chim. (Paris) 3, 49 (1968).

16. Szymanski, H., Salinas, C., and Kwitowski, P., Nature 188, 403 (1960).

17. Andrew, T. D., Phillips, C. S. G., and Semlyen, J. A., J. Gas Chromatog. 1, 27 (1963).

18. Brunst, W., "Die Induktive Wärmebehandlung," Springer, Berlin, 1962.

19. Bozorth, R. M., "Ferromagnetism," Van Norstrand Company, Toronto, New York, London, 1951.

20. Bühler, Ch., Thesis ETH, Zürich, 1970. 

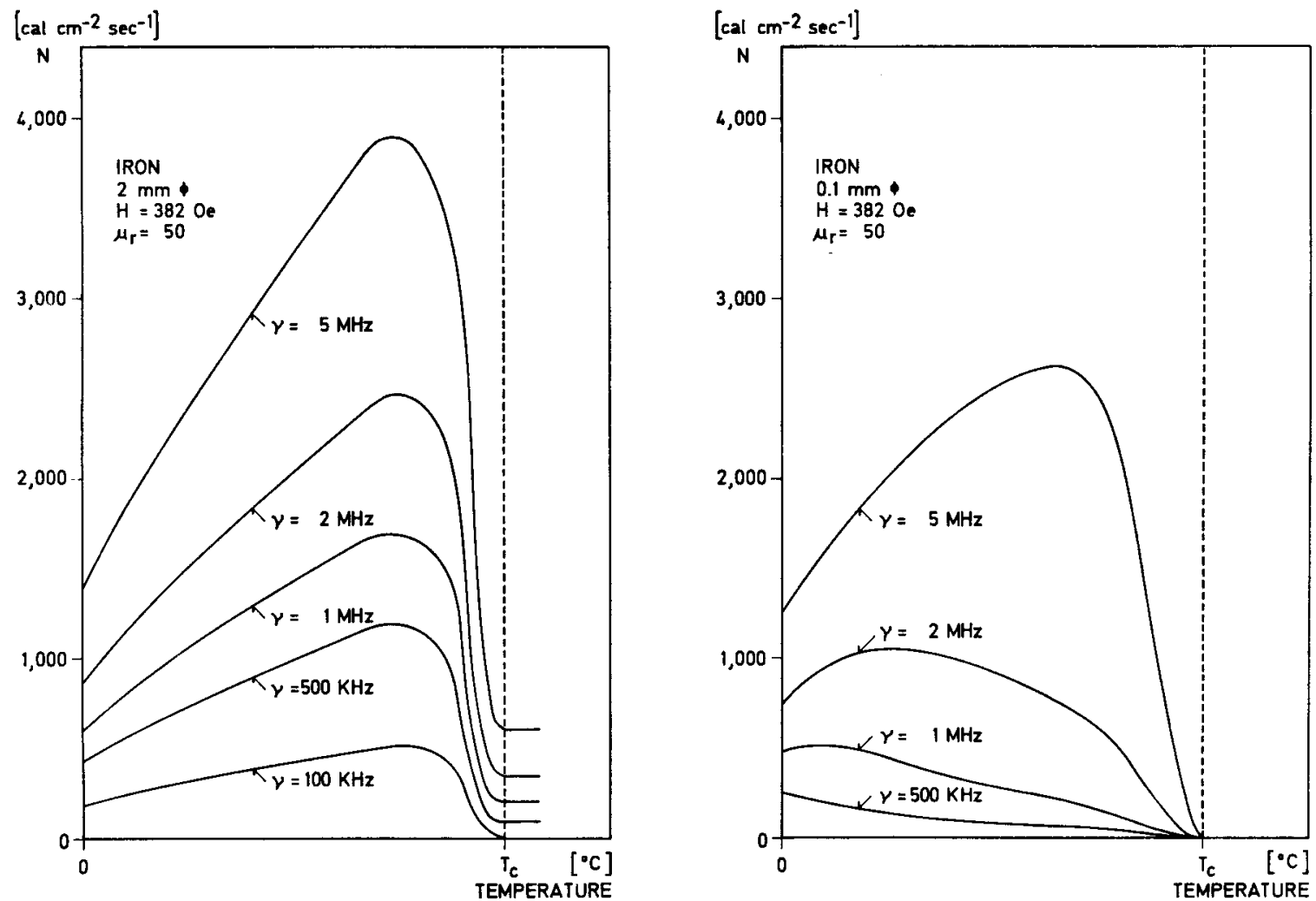

Figure 2. Temperature dependence of the power consumption of iron conductors in a high frequency induction field of 328 Oe as a function of the oscillator frequency.
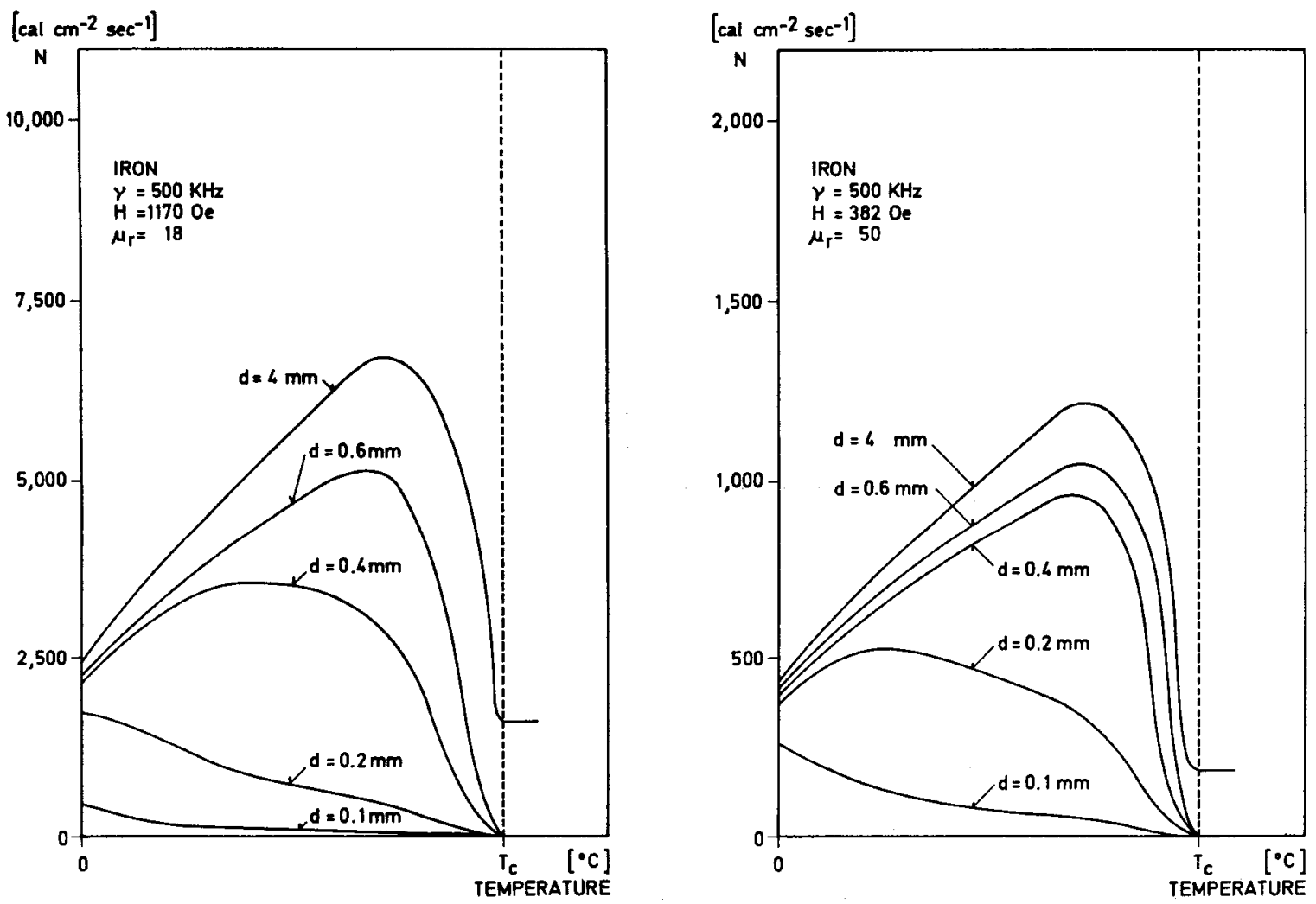

Figure 3. Temperature dependence of the power consumption of iron conductors in a high frequency induction of 1170 and 382 $\mathrm{Oe}$ as a function of the diameter of the conductor at an oscillat or frequency of $500 \mathrm{KHz}$.

was calculated. On inspection of these figures it becomes obvious that there is only a small change in power consumption at the Curie Point $T_{c}$ for wire diameters of approximately $0.1 \mathrm{~mm}$ and frequencies up to around $1 \mathrm{MHz}$. Wire diameters of more than 1 mm give a relatively high power consumption above the Curie Point $T_{c}$; this results in comparatively small heat losses and concurrently higher end temperatures, which may lie above $T_{c}$. Considering radiation and conduction heat losses warm up curves as given in Figures 

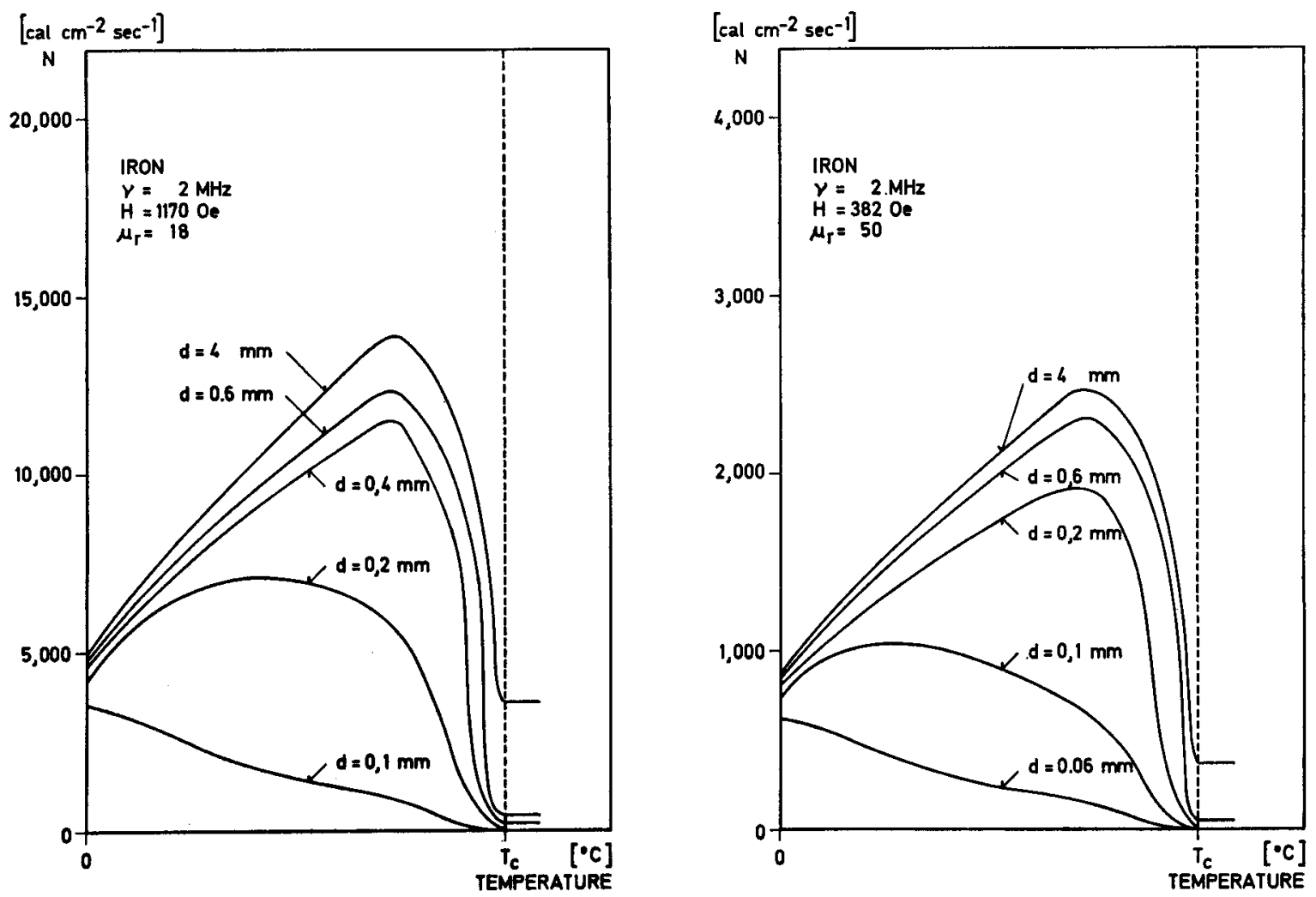

Figure 4. Temperature dependence of the power consumption of iron conductors in a high frequency induction field of 1170 and $382 \mathrm{Oe}$ as a function of the diameter of the conductor at an oscillator frequency of $2 \mathrm{MHz}$.

Table I. Data for Different Oscillators Used in PGC.

\begin{tabular}{|c|c|c|c|c|c|c|}
\hline \multirow{2}{*}{$\begin{array}{l}\text { Type of } \\
\text { Oscillator }\end{array}$} & \multirow{2}{*}{$\begin{array}{c}\text { Frequency of } \\
\text { Oscillator } \\
v \\
{[\mathrm{KHz}]}\end{array}$} & \multirow{2}{*}{$\begin{array}{c}\text { Applied } \\
\text { Magnetic } \\
\text { Field } \\
\text { [Oe] }\end{array}$} & \multicolumn{3}{|c|}{$\begin{array}{c}\text { Relative Permeability } \\
\mu_{\mathrm{r}}\left[\mathrm{O}^{\circ} \mathrm{C}\right]\end{array}$} & \multirow{2}{*}{$\begin{array}{l}\text { Rise-time of } \\
\text { an iron wire } \\
\text { of } 0.5 \mathrm{~mm} \\
\text { diam. to Curie } \\
\text { temperature [sec }\end{array}$} \\
\hline & & & $\mathrm{Fe}$ & $\mathrm{Ni}$ & Co & \\
\hline $\mathrm{ETH}^{(7)}$ & 480 & 1170 & 18 & 13.5 & 11.6 & $30 \cdot 10^{-3}$ \\
\hline Fischer* & 1200 & 382 & 50 & 16.5 & 21 & $80 \cdot 10^{-3}$ \\
\hline Pye Unicam** & 550 & - & - & - & - & ca. 2 \\
\hline Packard*** & - & - & - & - & - & - \\
\hline
\end{tabular}

*Hochfrequenz-Pyrolyse, Fischer Labortechnik, 532 Bad Godesberg, Germany.

** Curie Point Pyrolyser, Pye Unicam Ltd., Cambridge, England.

***Model 891, Packard Instrument Company, Inc., Downers Grove, Ill.

5 and 6 can be computed (21). These figures show that the optimal wire diameter for a fast warm-up and a constant end temperature decreases with increasing frequency and has to be defined for each and every oscillator used. For oscillators of about $500 \mathrm{KHz}$ and $1.2 \mathrm{MHz}$ the wire diameters should be around 0.5 and $0.2 \mathrm{~mm}$, respectively. Since the magnetic Field $\mathrm{H}$ within the coil depends on the output of the oscillator and the induction coil geometry, these two parameters are important for the rise-time (see Equation 1).

The computed rise times shown in Figures 5 and 6 are in perfect agreement with experimental values $(7,22,23)$. An example of a warm-up of an iron conductor is given in Figure 7. It was recorded using a low-mass thermocouple and an oscilloscope as de- scribed earlier (22). A low generator output obviously leads to small magnetic fields $\mathrm{H}$ and long rise-times $(2,13)$. This, as well as unsuitable choice of conductor diameters and improper coupling of the coil to the oscillator, may have been the cause for some of the relatively long rise-times observed (2). In contrast to certain claims (14) there are only small differences in the temperature rise-times between the surface and center of a conductor at frequencies around $500 \mathrm{KHz}$

21. Schuster, R., Diss. Ilmenau, 1966.

22. Giacobbo, H., Diss. ETH, Zürich, 1964.

23. Simon, W., Kriemler, P., Voellmin, J. A., and Steiner, H., J. Gas Chromatog. 5, 53 (1967). 

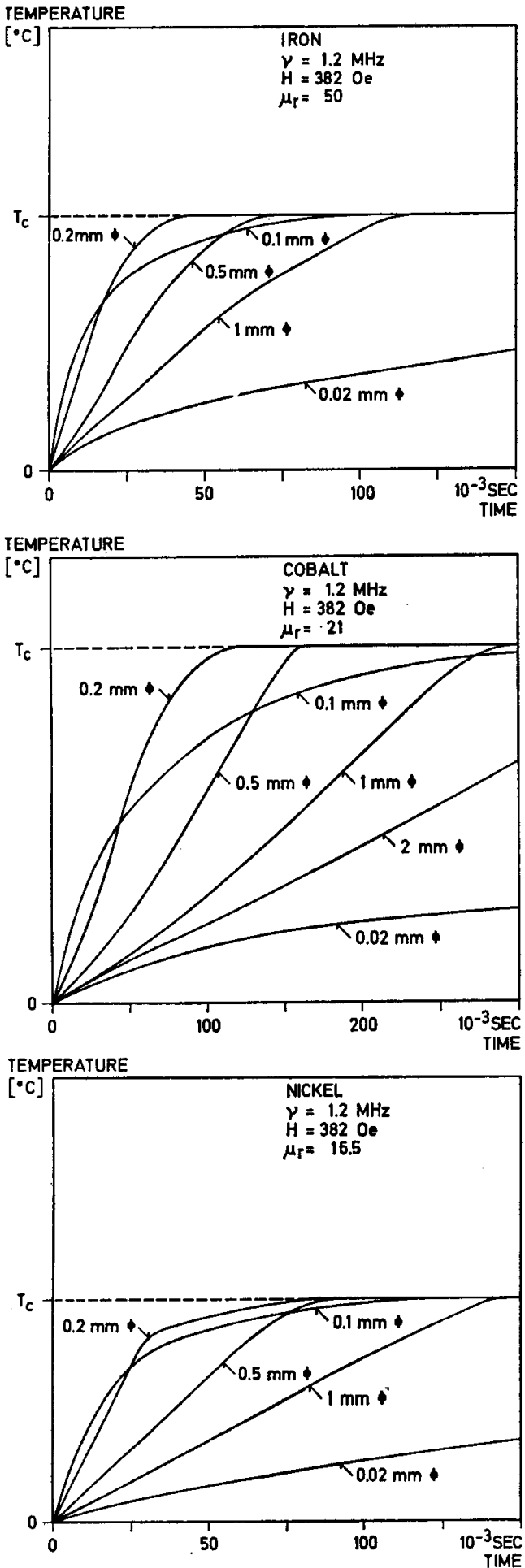

Figure 5. Temperature-Time profiles of iron, cobalt and nickel conductors of different diameters in a high frequency induction field of $382 \mathrm{Oe}$ and $1.2 \mathrm{MHz}$.

(24). There are therefore no basic limitations to using ferromagnetic tubes in Curie Point pyrolysis and placing the sample to be studied within this tube [see $\mathrm{L}$. Ettre in (4) and Figure 8].

F. Farré-Rius and G. Guichon (5) point out that there are polymers showing decomposition half-times at $600^{\circ} \mathrm{C}$ smaller than the rise-time of about $30 \cdot 10^{-3} \mathrm{sec}$ of a typical Curie Point pyrolyzer so that most of the sample will be fragmented before the temperature equilibrium of the pyrolysis system is reached. For
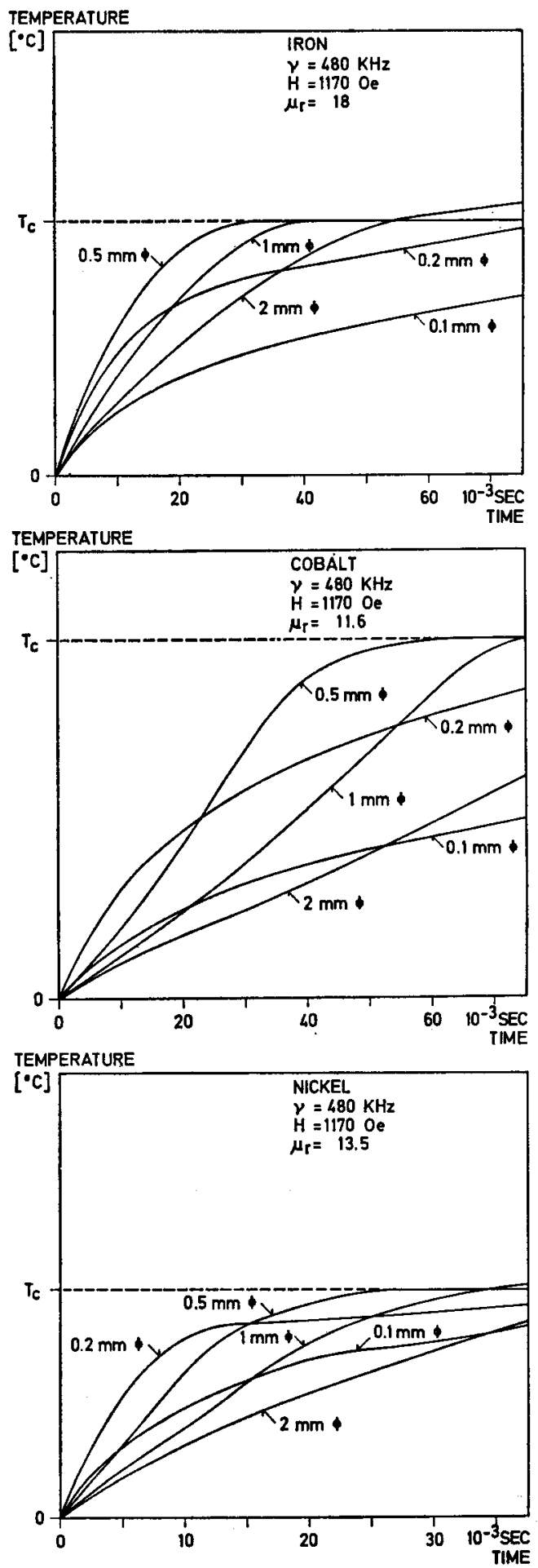

Figure 6. Temperature-Time profiles of iron, cobalt and nickel conductors of different diameters in a high frequency induction field of $1170 \mathrm{Oe}$ and $480 \mathrm{KHz}$.

such cases obviously either an alloy with a lower Curie Point (lower pyrolysis temperature) * or a faster warm

*Ferromagnetic materials with Curie-Points of 400, 500, $600,700,800^{\circ} \mathrm{C}$ (Hochfrequenz-Pyrolyse, Fischer Labortecknik, 532 Bad Godesberg, Germany) as well as many other materials for the range $70-1000^{\circ} \mathrm{C}$ are available ( 7$)$.

24. Hegewald, F., Brown-Boveri Company Nachrichten 434 (July/August) 1961. 


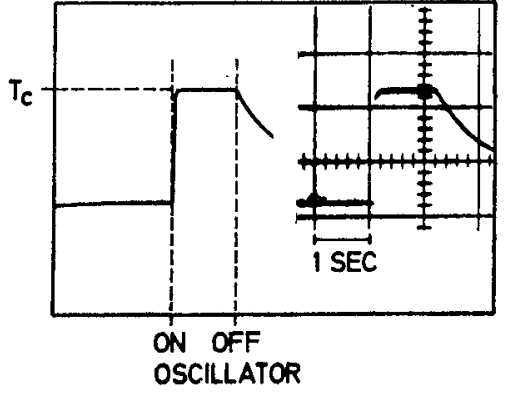

Figure 7. Temperature-Time profile of an iron conductor of $0.6 \mathrm{~mm}$ diameter in a high frequency induction field of 1170 Oe and $480 \mathrm{MHz}$. Left: schematic diagram; Right: Oscilloscope trace.

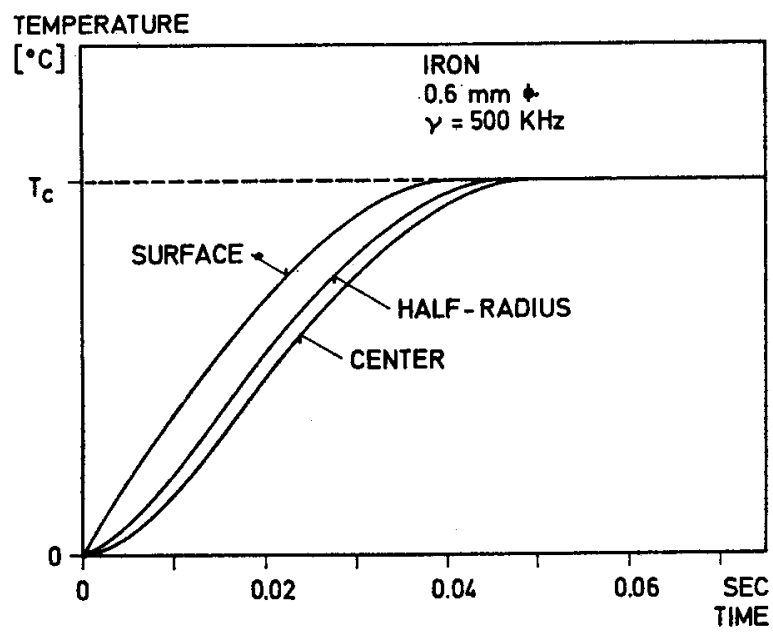

Figure 8. Computed warm-up of an iron wire in high frequency induction field.

up has to be chosen. At constant magnetic fieldstrength inside the high frequency induction coil the temperature rise-time may be substantially decreased by using larger oscillator frequencies and/or smallerdiameter ferromagnetic conductors. At $10 \mathrm{MHz}$ the risetime of an iron wire of $0.05 \mathrm{~mm}$ diameter is reduced to $5 \cdot 10^{-3}$ sec. $(3820$ e).

Although different systems giving short filament risetimes have been described recently (2), there are uncertainties as to the end temperatures attained which is not the case with Curie Point systems. Table II shows results obtained in the determination of the Curie Point of materials of different origin. The values given in column two were obtained using a thermobalance (Mettler TA1, Mettler Instruments AG, CH8606 Greifensee-Zürich, Switzerland) and a permanent magnet a short distance from the sample pen. The temperature $T_{c}{ }^{*}$ of maximum weight change (dW/ dT) $)_{\max }$ given there is around $15^{\circ} \mathrm{C}$ lower than the temperature for $(\mathrm{dW} / \mathrm{dT})=0$. Samples with widely differing histories give identical measurements; as Table II shows, $\mathbf{T}_{\mathrm{c}}^{*}$ is $745 \pm 5^{\circ} \mathrm{C}$, which is within the uncertainty-limits. A repeated determination of the end temperature of a wire of $0.5 \mathrm{~mm}$ diameter using different oscillators gave $765 \pm 5^{\circ} \mathrm{C}$, which is in good agreement with published data on Curie temperatures (Table III)
Table II. Curie Temperature $T_{c}{ }^{*}$ of Iron Wires of Different Origin.

Wire Diameter

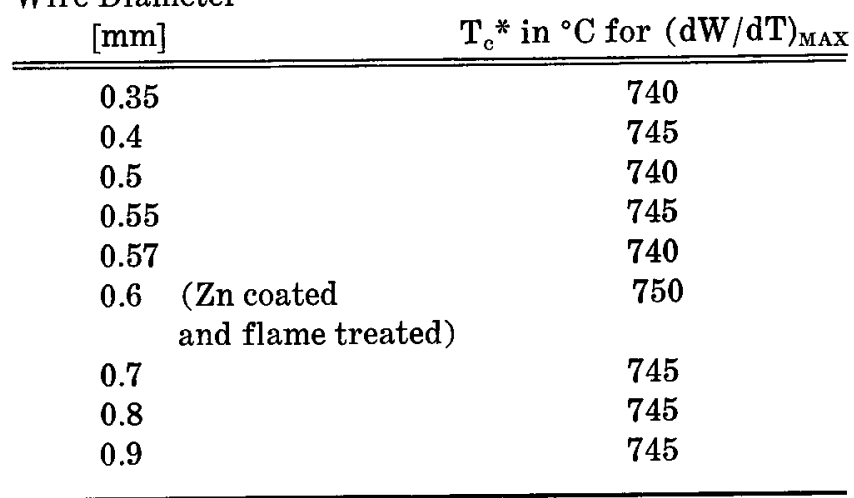

Table III. Curie Temperatures $T_{\mathrm{c}}$.

\begin{tabular}{lr} 
Metal & $\mathrm{T}_{\mathrm{e}}\left[{ }^{\circ} \mathrm{C}\right]$ \\
\hline \hline Nickel & 358 \\
Iron & 770 \\
Cobalt & 1128
\end{tabular}

Table IV. Distribution of Radioactivity after the Pyrolysis of $100 \mu \mathrm{g}$ Labelled Sodium Salt of Benzoic Acid.

\begin{tabular}{|c|c|c|}
\hline \multirow{2}{*}{$\begin{array}{c}\text { Source of Radioactivity } \\
\text { after Pyrolysis }\end{array}$} & \multicolumn{2}{|c|}{$\begin{array}{c}\text { Activity }[\%] \text { out } \\
\text { of Benzoic Acid Salt }\end{array}$} \\
\hline & Ring $-{ }^{-14} \mathrm{C}$ & ${ }^{-14} \mathrm{COO} \theta$ \\
\hline Ferromagnetic conductor & 7 & 26 \\
\hline $\begin{array}{l}\text { Pyrolysis glass capillary } \\
\text { (around conductor) }\end{array}$ & 44 & 48 \\
\hline $\begin{array}{l}\text { Components eluted from } \\
\text { GC column }\end{array}$ & 35 & 23 \\
\hline Losses & 14 & 3 \\
\hline
\end{tabular}

as well as the temperature of about $760^{\circ} \mathrm{C}$ for (dW/ $\mathrm{dT})=0$.

Although short temperature rise-times as well as constant and reproducible end temperatures may easily be obtained, the decrease in temperature after turning off the power is slow in comparison to the initial temperature rise (Figure 9). In Figure 9 the computed decrease in temperature of wires of $2 \mathrm{~cm}$ length are given as a function of time. The lowest curve was computed for a wire having an electric connection to allow the temperature to be followed by resistance measurement. The agreement with experimental values (circles) is obvious. Since after pyrolysis a large percentage of the pyrolysed material and the sample may remain on the conductor as well as on the glass walls surrounding the wire, a slow decrease in temperature must lead to irreproducible pyrolysis gas chromatograms (see Table IV). Conductors of smaller diameter (around $0.05 \mathrm{~mm}$ ) and therefore smaller mass may 


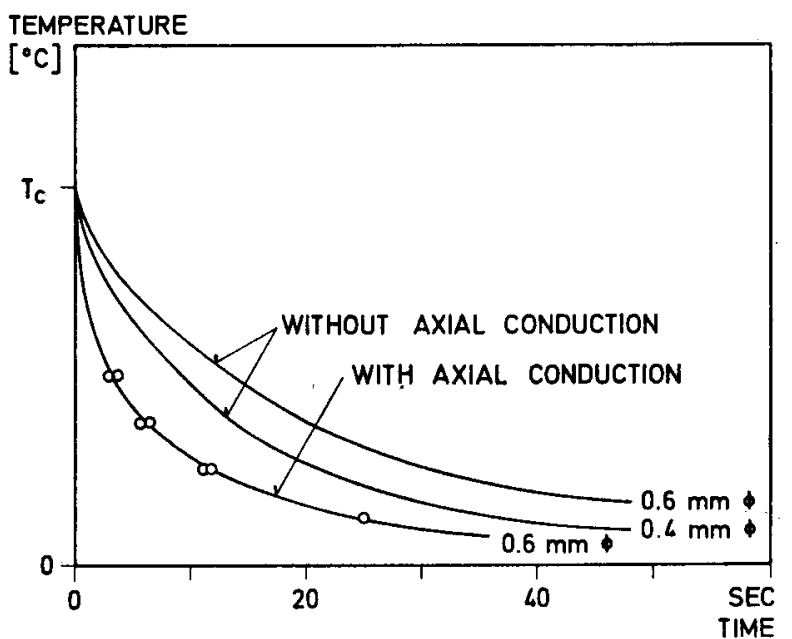

Figure 9. Decrease in temperature of iron wires after warm-up to the Curie temperature.

give almost ideal square-wave temperature-time profiles if adequate oscillator frequencies are used (1). The problems connected with the slow decrease in temperature of conventional Curie Point pyrolysis sys- tems may easily be overcome by by-passing the pyrolysis zone after turning off of the oscillator or by pyrolysing the sample in ferromagnetic tubes as will be shown later.

A detailed study of the surface of sample supports used in the pyrolysis GC by electron microscopy has shown that a control of the surface is of utmost importance to improve reproducibility. Details will be given elsewhere.

\section{Acknowledgment}

We thank Mettler Instruments AG, CH-8606 Greifensee-Zürich, Switzerland, for the determination of Curie temperatures by thermogravimetry. The present work has been supported by the Schweizerischer Nationalfonds zur Foerderung der wissenschaftlichen Forschung (Research Project No. 5188.2).

Manuscript received March 20, 1970

Presented at the Sixth International Symposium on Advances in Chromatography held in Miami Beach, Florida, June 2-5, 1970. 